\title{
Using Feedback Control of Microflows to Independently Steer Multiple Particles
}

\author{
Michael D. Armani, Satej V. Chaudhary, Roland Probst, and Benjamin Shapiro, Member, IEEE
}

\begin{abstract}
In this paper, we show how to combine microfluidics and feedback control to independently steer multiple particles with micrometer accuracy in two spatial dimensions. The particles are steered by creating a fluid flow that carries all the particles from where they are to where they should be at each time step. Our control loop comprises sensing, computation, and actuation to steer particles along user-input trajectories. Particle locations are identified in real-time by an optical system and transferred to a control algorithm that then determines the electrode voltages necessary to create a flow field to carry all the particles to their next desired locations. The process repeats at the next time instant. Our method achieves inexpensive steering of particles by using conventional electroosmotic actuation in microfluidic channels. This type of particle steering does not require optical traps and can noninvasively steer neutral or charged particles and objects that cannot be captured by laser tweezers. (Laser tweezers cannot steer reflective particles, or particles where the index of refraction is lower than (or for more sophisticated optical vortex holographic tweezers does not differ substantially from) that of the surrounding medium.) We show proof-of-concept PDMS devices, having four and eight electrodes, with control algorithms that can steer one and three particles, respectively. In particular, we demonstrate experimentally that it is possible to use electroosmotic flow to accurately steer and trap multiple particles at once.

[1541]
\end{abstract}

Index Terms-Electroosmotic actuation, electrophoretic, feedback control, microfluidics, particles, steering, trapping.

\section{INTRODUCTION}

$\mathbf{T}$ HE ability to steer individual particles inside microfluidic systems is useful for navigating particles to localized sensors, for cell sorting, for sample preparation, and for combinatoric testing of particle interactions with other particles, with chemical species, and with distributed sensors. A variety of methods are currently used to manipulate particles inside microfluidic systems: individual particles can be steered by laser tweezers [1]-[3]; they can be trapped, and steered to some degree, by dielectrophoresis (DEP) [4]-[7]; and by traveling-wave-dielectrophoresis (TWD) [7], [8]; held by acoustic traps [9]; steered by manipulating magnets attached to the particles [10]; and guided by a MEMS pneumatic array [11]. There is also a feedback control approach (similar to the one developed in this paper) used by Cohen [12], [13] to trap and steer a single particle, but not yet multiple particles, using electroosmotic or electrophoretic actuation.

Manuscript received March 10, 2005; revised February 1, 2006. Subject Editor C. Liu.

M. D. Armani, S. V. Chaudhury, and R. Probst are with the Department of Aerospace Engineering, University of Maryland, College Park, MD 20742 USA.

B. Shapiro is with the Department of Aerospace Engineering and the Bio-Engineering Department, University of Maryland, College Park, MD 20742 USA (e-mail: benshap@eng.umd.edu).

Digital Object Identifier 10.1109/JMEMS.2006.878863
Of these methods, laser tweezers are the gold standard for single particle manipulation. Ashkin's survey article [1] provides a history of optical trapping of small neutral particles, atoms, and molecules. Current laser tweezer systems can create up to 400 three-dimensional (3-D) traps, they can trap particles ranging in size from tens of nanometers to tens of micrometers, trapping forces can exceed $100 \mathrm{pN}$ with resolutions as fine as $100 \mathrm{aN}$, and the positioning accuracy can be below tens of nanometers [2], [14]. However, optical tweezers require lasers and delicate optics and the whole system is unlikely to be miniaturized into a handheld format. The other methods aforementioned (DEP, acoustic traps, manipulation via attached magnets, and steering via pneumatic arrays systems) can be miniaturized into handheld formats but their steering capabilities are not as sophisticated as those of laser tweezers.

Our approach uses vision-based microflow control to steer particles by correcting for particle deviations - at each time we create a fluid flow to move the particles from where they are to where they should be. This allows very simple devices, actuated by routine methods, to replicate the planar steering capabilities typically requiring laser tweezers. We have shown that our approach permits a PDMS device with four electrodes to steer a single cell, and a device with eight electrodes to steer up to three particles simultaneously. The method is noninvasive (the moving buffer simply carries the cells along), the entire system can be miniaturized into a handheld format (both the control algorithms and the optics can be integrated onto chips), we can steer almost any kind of visible particle (neutral particles are carried along by the electroosmotic flow, charged particles are actuated by a combination of electroosmosis and electrophoresis), and the system is cheap (the most expensive part is the camera and microscope, and these will be replaced by an on-chip optical system for the next generation of devices).

Due to the correction for errors provided by the feedback loop, the flow control algorithm steers the particles along their desired paths even if the properties of the particles (their charge, size, and shape) and the properties of the device and buffer (the exact geometry, the zeta potential, $\mathrm{pH}$, and other factors) are not known precisely. The fundamental disadvantage of our approach is its lower accuracy as compared to laser tweezers: our positioning accuracy will always be limited by the resolution of the imaging system and by the Brownian motion that particles experience in-between flow control corrections. Our current optical resolution is on the order of $1 \mu \mathrm{m}$, and the particle Brownian drift during each control time step is less than $80 \mathrm{~nm}$.

Both feedback and microflows are essential for our particle steering capability. Feedback is required to correct for particle position errors at each instant in time. Microfluidics is required because macroflows exhibit more complex dynamics, due to 


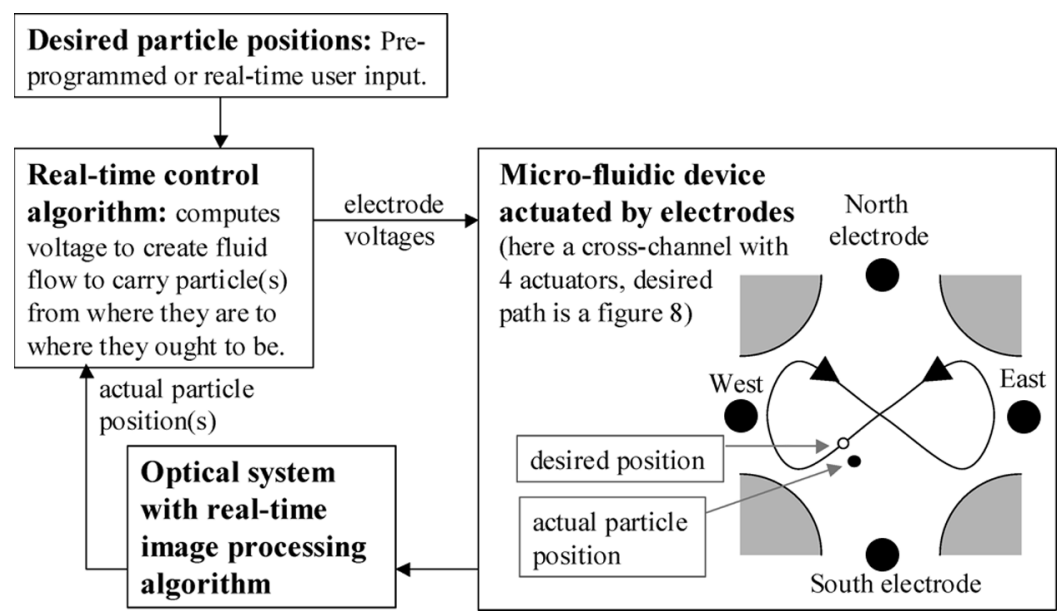

Fig. 1. Feedback control particle steering approach for a single particle. A microfluidic device with standard electroosmotic actuation is observed by a vision system that informs the control algorithm of the current particle position. The control algorithm compares the actual position against the desired position and finds the actuator voltages that will create a fluid flow, at the particle location, to steer the particles from where it is to where it should be. The process repeats continuously to steer the particle along its desired path.

their momentum effects, and it is not possible to find the external actuator inputs that will reliably create macroflows to steer particles. On the microscale, the Stokes equations can be inverted to determine the necessary actuation that will steer many particles at once.

This paper presents a proof-of-concept: we show experimentally that it is possible to steer up to three particles at once using a simple eight electrode PDMS device. The paper is organized as follows: Section II provides an overview of the feedback particle steering method, device fabrication is discussed in Section III, experimental methods for system operation are described in Section IV, the control algorithms are summarized in Section V, and experimental particle steering results are presented in Section VI.

\section{Overview of Steering by FeEdback Flow Control}

Fig. 1 shows the basic control idea for a single particle: a microfluidic device, an optical observation system, and a computer with a control algorithm, are connected in a feedback loop. The vision system locates the position of the particle in real time, the computer then compares the current position of the particle with the desired (user input) particle position, the control algorithm computes the necessary actuator voltages that will create the electric field, or the fluid flow, that will carry the particle from where it is to where it should be, and these voltages are applied at electrodes in the microfluidic device. For example, if the particle is currently north/west of its desired location, then a south/east flow must be created. The process repeats at each time instant and forces the particle to follow the desired path (see also, [15]).

Both neutral and charged particles can be steered in this way: a neutral particle is carried along by the flow that is created by electroosmotic forces, a charged particle is driven by a combination of electroosmotic and electrophoretic effects. In either case, it is possible to move a particle at any location to the north, east, south, or west by choosing the appropriate voltages at the four electrodes. It is also possible to use this scheme to hold a particle in place: whenever the particle deviates from its desired position, the electrodes create a correcting flow to bring it back to its target location.

Surprisingly, it is also possible to steer multiple particles independently using this feedback control approach [16]. A multielectrode device is able to actuate multiple fluid flow or electric field modes. Different modes cause particles in different locations to move in different directions. By judiciously combining these modes, it is possible to move all particles in the desired directions.

The development of a control algorithm that can combine modes in this manner is our key theoretical contribution: this algorithm is described in detail in [16] and is summarized here in Section V-C. The algorithm requires some knowledge of the particle and system properties (charged particles exhibit electrophoresis and react differently than neutral particles) but this knowledge does not have to be precise: the reason is that feedback, the continual comparison between the desired and actual particle positions, serves to correct for errors and makes the system robust to experimental uncertainties [17], [18]. Even though our experiments have sources of error, some of which are unavoidable, such as variations in device geometry, parasitic pressure forces caused by surface tension at the reservoirs, Brownian noise, and variations in zeta potentials and charges on the particles-our control algorithm still steers all the particles along their desired trajectories.

\section{SYSTEM FABRICATION}

We describe the fabrication of the four- and eight-electrode PDMS devices for single and multiparticle steering.

\section{A. Fabrication of the Four Electrode PDMS Device}

The microfluidic devices were fabricated using the soft lithography steps described in [19]. Fig. 2 shows the fabrication sequence for the PDMS device of Fig. 3 that was used to steer a single particle by electroosmotic flow control.

The masks for the device were designed using LEDIT software (Tanner EDA) and exported as GDS file-types. 
$\mathrm{Si}$

1. Dehydrate silicon wafer
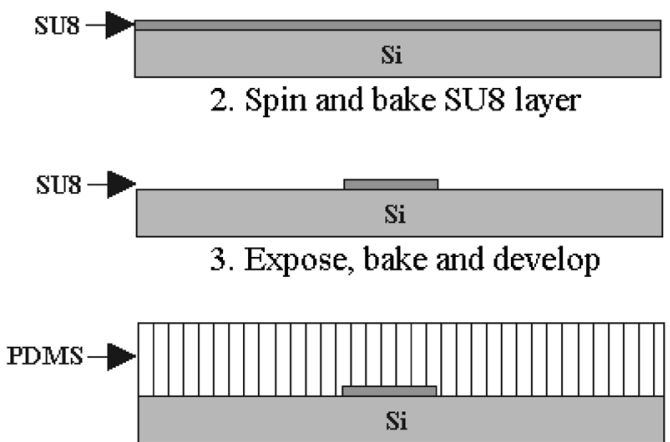

4. Create replica using PDMS

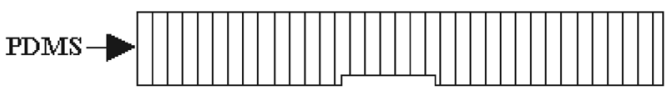

5. Peel off PDMS replica

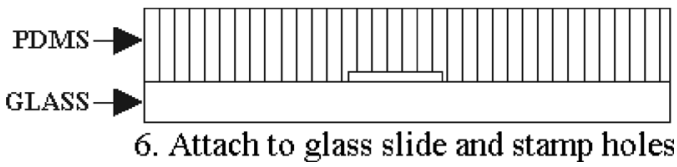

Fig. 2. Fabrication sequence for the microfluidic particle steering PDMS devices.
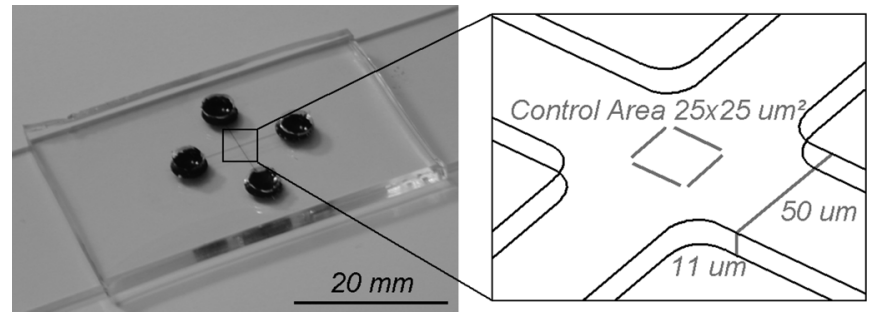

Fig. 3. Left: photograph of the PDMS on glass device filled with blue coloring $(1 \%$ Methylene Blue $(\mathrm{aq}))$ to clearly show the microfluidic channels and reservoirs. The two channels are $2 \mathrm{~cm}$ long, $50 \mu \mathrm{m}$ wide, and $11 \mu \mathrm{m}$ deep. Right: schematic of the channel intersection and the $25 \mathrm{~mm} \times 25 \mathrm{~mm}$ particle steering control area. In this device, the roughness was measured by a profilometer to be $59.2 \mathrm{~nm}$ for the PDMS top covering and $27.8 \mathrm{~nm}$ for the bottom glass cover slip.

Chromium/glass masks were then obtained from Berkeley MicroLab (which used a GCA Mann 3600 Pattern Generator and mask developer to produce 5 -in wafers with features accurate to $10 \mathrm{~nm}$ ).

An SU8 master template was created on a silicon wafer. The 100-mm basic grade, single side silicon wafers were obtained from UniversityWafer. The wafers were baked at $200^{\circ} \mathrm{C}$ for $1 \mathrm{~h}$ to dehydrate the wafer surface, $4 \mathrm{ml}$ of SU8-5 (Microchem) was deposited on the polished side of the wafer and was spin coated at $500 \mathrm{rpm}$ for $5 \mathrm{~s}$ and then $1100 \mathrm{rpm}$ for $30 \mathrm{~s}$. This was followed by a softbake $\left(95^{\circ} \mathrm{C}\right.$ for $\left.30 \mathrm{~min}\right)$, UV exposure $\left(650 \mathrm{~mJ} / \mathrm{cm}^{2}\right)$, post-bake $\left(95^{\circ} \mathrm{C}\right.$ for $\left.30 \mathrm{~min}\right)$, and development in SU8 developer (Microchem). The wafer was rinsed in liberal amounts of isopropanol, methanol, and deionized water and blowdried with nitrogen.
The next step was the creation of PDMS microchannels by replication molding. Ten-part silicone elastomer (Sylgard 184, Dow Corning) was mixed with one-part curing agent (Sylgard 184, Dow Corning) and poured over the silicon wafer, which was contained in a large Petri dish. The PDMS mixture was poured to a height of $3 \mathrm{~mm}$ above the wafer surface. The PDMS was cured at room temperature for $24 \mathrm{~h}$. A razor blade was then used to cut out the section of PDMS containing the channels, and tweezers were used to peel off the PDMS with the microchannels facing down.

A $3 \times 1$-in microscope slide (Fisher Scientific) was used as a bottom surface for the 4-electrode devices and a 4-in diameter Pyrex wafer (Mark Optics) was used for the larger 8-electrode devices. We used a simple PDMS/glass bonding technique described in [20] and [21]. The PDMS was pressed on top of the glass substrates, it conformed to minor imperfections in the glass and bonded to it by weak van der Waal forces [20], creating a reversible bond and a watertight seal. As noted in [21], sealing was fast, occurred at room temperature, and would withstand fluid pressures up to 5 psi.

Holes were stamped in the PDMS using a 10-mm cork borer (McMaster Carr) for electrical and fluid access to the channels. The devices were filled with deionized water as described in Section IV. Wire electrodes (30-gauge platinum wire, Surepure Chemetals, Inc.) were inserted into the four holes by hand. The platinum electrode material was chosen because it prevents electrode erosion, which occurs through electrolysis.

A photograph of the PDMS device and a schematic view of the cross-channel particle-control region is shown in Fig. 3. The small $11 \mu \mathrm{m}$ channel depth and the large reservoir geometry of the device were chosen to minimize the effect of parasitic surface-tension-driven pressure flows, which act as flow errors, compared to the desired electroosmotic flow control velocities.

\section{B. Fabrication of the Eight Electrode PDMS Device}

The fabrication of the eight electrode devices was similar to the four electrode devices but with modifications based on lessons we learned from steering a single particle.

The size of the reservoirs was increased, and the electrodes were moved further away from the entry of the channel into the reservoir to decrease the effect of electrochemical phenomena (such as electrolysis and acid/base fronts that originate at the electrodes [22]) on flow in the channels. To fit the larger 8-reservoirs geometry we used a 4-in diameter Pyrex glass wafers instead of the $3 \times 1$-in microscope glass slides. PDMS reservoirs were fabricated, as opposed to stamped, by including the reservoir shapes in the SU8 master template thereby creating more repeatable device geometries. Access holes to the reservoirs were still created by stamping. The channels lengths were shortened to $7 \mathrm{~mm}$ so that a lower voltage would create the same electric field and flow velocity in the central control chamber.

\section{EXPERIMENTAL METHODS}

Here we describe the materials used in the experiment, the actuation method, and the vision system that is used to track the particles in real time. The control algorithm for steering a single particle is described in Section V-B and the algorithm for multiple particles is summarized in Section V-C. 


\section{A. Materials Used}

The device, glass, and electrode materials are described in Section III. For all experiments we used deionized water (J.T. Baker HPLC grade) with resistivity $1.25 \mathrm{M} \Omega \mathrm{cm}$ (measured using a Keithley 2400 Source Meter) and pH of $6.0 \pm 0.25$ (as measured by Fisher Liquid Universal $\mathrm{pH}$ Indicator [pH measurement range 4-10]). Ultrapure deionized water is expected to have a $\mathrm{pH}$ of 7.0 and resistivity of $18.0 \mathrm{M} \Omega \mathrm{cm}$, but exposure to carbon dioxide in air typically results in a lowered $\mathrm{pH}$ of 5.7 and resistivity of about $1.0 \mathrm{M} \Omega \mathrm{cm}$ [23].

For steering of cells, we incubated bakers yeast (Red Star, Giant Food) for $24 \mathrm{~h}$ in sugary water (30 $\mathrm{mg}$ glucose per milliliter). To make a single-cell suspension, we filtered the yeast solution using a $10-\mu \mathrm{m}$ polyester filter (Fisher Scientific). This filtered yeast solution was added to the deionized water at $10 \mathrm{mg} / \mathrm{ml}$. To prevent cell adhesion to solid surfaces, channels were filled with $20 \mathrm{mg} / \mathrm{ml}$ bovine serum albumin (BSA) (Sigma-Aldrich), left for $30 \mathrm{~min}$, and flushed five times with ethanol. We also added $1 \mathrm{mg} / \mathrm{ml} \mathrm{BSA}$ to the buffer solution to prevent cells from sticking to each other and to replenish antistick surface coatings during the particle steering experiments.

For steering of beads, we used either Polysciences brand polystyrene beads (diameter $5 \mu \mathrm{m} \pm 0.975 \mu \mathrm{m}$ standard deviation) or Duke Scientific fluorescent polystyrene beads (diameter $2.2 \pm 0.11 \mu \mathrm{m})$. Bead solution was added to deionized water to achieve bead concentrations that would yield just a few beads in the control chambers. No BSA pretreatment or addition was necessary for experiments using beads.

\section{B. Electroosmotic Flow Actuation and Particle Velocities}

Platinum electrodes inserted into the four or eight reservoirs actuate the fluid flow. The voltage on the electrodes is set by the control algorithm (Section V) running on a personal computer (Dell Precision Workstation 530, Xeon 1.7 GHz, 2 GB memory, WinXP), is sent to a digital-to-analog signal converter (National Instruments DAQ), and is then passed to a 17-channel operational amplifier (APEX). For the two experiments, we used 4 and 8 out of the 17 available amplifier channels with a range of \pm 30 and $\pm 10 \mathrm{~V}$, respectively (the eight electrode device had shorter channels and required less voltage). The resulting electric fields create electroosmotic flow in the device and the flow velocity is given by [24], [25]

$$
\vec{V}(x, y)=\frac{\varepsilon \zeta}{\eta} \vec{E}(x, y)
$$

where $\vec{E}$ is the local electric field, it varies in the $x, y$ directions and is uniform in the vertical $z$ direction, $\varepsilon$ is the permittivity of the liquid, $\eta$ is its dynamic viscosity, and $\zeta$ is the zeta potential at the liquid/solid interface. We measured the value of our electroosmotic mobility by a current monitoring technique (as in [26]), and found $\vec{V} / \vec{E}=u=(36.5 \pm 3.6) \times$ $10^{-9} \mathrm{~m}^{2} \mathrm{~V}^{-1} \mathrm{~s}^{-1}$ which is in good agreement with values of $36 \times 10^{-9} \mathrm{~m}^{2} \mathrm{~V}^{-1} \mathrm{~s}^{-1}$ and $40 \times 10^{-9} \mathrm{~m}^{2} \mathrm{~V}^{-1} \mathrm{~s}^{-1}$ reported for PDMS/glass channels at neutral pH [26], [27]. Our zeta potential $\zeta$ followed from (1) above which, for water at $25^{\circ} \mathrm{C}$, yielded $\zeta=46.9 \pm 7.6 \mathrm{mV}$.
Particles are carried along by the electroosmotic flow, but charged particles also experience electrophoretic velocities. In the literature, electrophoretic mobilities have been reported for $50 \mathrm{~nm}$ to $1 \mu \mathrm{m}$ diameter latex beads $(c=-45$ to $\left.-79 \times 10^{-9} \mathrm{~m}^{2} \mathrm{~V}^{-1} \mathrm{~s}^{-1}\right)$, for bacteria $(-3.3$ to $-45 \times$ $\left.10^{-9} \mathrm{~m}^{2} \mathrm{~V}^{-1} \mathrm{~s}^{-1}\right)$, yeast $\left(-11\right.$ to $\left.-31 \times 10^{-9} \mathrm{~m}^{2} \mathrm{~V}^{-1} \mathrm{~s}^{-1}\right)$, endothelial cells $\left(-7.4 \times 10^{-9} \mathrm{~m}^{2} \mathrm{~V}^{-1} \mathrm{~s}^{-1}\right)$, erythrocyte cells $\left(-10.3 \times 10^{-9} \mathrm{~m}^{2} \mathrm{~V}^{-1} \mathrm{~s}^{-1}\right)$, and lymphocyte cells $\left(-25.3 \times 10^{-9} \mathrm{~m}^{2} \mathrm{~V}^{-1} \mathrm{~s}^{-1}\right)$ [28]-[35]. Our beads and cells acquire a surface charge depending on their surrounding $\mathrm{pH}$, temperature, the concentration of the particles, and the type of impurities in the medium [24], [36].

We do not rigorously control $\mathrm{pH}$, temperature, concentration, and impurities in our simple devices and this makes it difficult for us to measure electrophoretic mobilities reliably. (Recall that the steering algorithm does not need an accurate measurement of the particle mobilities. It works even if the mobilities are only known to within $\pm 50 \%$.) During the steering experiments, the net particle mobilities are first measured on-line as described in Section IV-D.

We have also measured mobilities independently off-line using devices with longer $(5.6 \mathrm{~cm})$ channels and applying a lower electric field $(48.3 \mathrm{~V}$ across $5.6 \mathrm{~cm}$ versus $10 \mathrm{~V}$ across $1.4 \mathrm{~cm}$ ) to limit, and keep the particles further away from, regions of electrochemistry. The $5.0 \mu \mathrm{m}$ polystyrene beads had a net (electroosmotic plus electrophoretic) mobility of $m=$ $u+c=(-20.8 \pm 2.0) \times 10^{-9} \mathrm{~m}^{2} \mathrm{~V}^{-1} \mathrm{~s}^{-1}$, the $2.2 \mu \mathrm{m}$ beads had $m=(-55.5 \pm 12.0) \times 10^{-9} \mathrm{~m}^{2} \mathrm{~V}^{-1} \mathrm{~s}^{-1}$, and the yeast cells had $m=(+13.2 \pm 3.3) \times 10^{-9} \mathrm{~m}^{2} \mathrm{~V}^{-1} \mathrm{~s}^{-1}$. This then gives a measurement of the electrophoretic mobility $c$ as $m-u$.

\section{Vision System to Locate Particles in Real Time}

The same vision system was used for both single and multiple particle tracking. It included a 40x magnification transmitted-light microscope (Nikon TS100); a 40 framesper-second, 480 by 640 gray-scale pixel camera (Vision Components, VC2038E DSP, Ettlingen, Germany); and a digitalsignal-processing (DSP) unit located inside the camera that evaluated the particle-tracking algorithm (described below). For steering of the fluorescent $2.2 \mu \mathrm{m}$ beads in the eight electrode devices, the vision system further included a bright 1 Watt LED light source [465 $\mathrm{nm}$ (blue), Luxeon], and a high-pass filter before the camera (480 $\mathrm{nm}$ and up, Chroma Technology Corporation), so that the beads, which emit light at $510 \mathrm{~nm}$ (green), were seen more clearly as green on black.

The image-processing algorithm runs on the DSP unit in the camera and tracks the location of all particles of interest. It is a combination of an algorithm that finds all particles in an image frame and an algorithm that tracks individual particles (see Fig. 5). A search window surrounds each particle that will be controlled. The algorithm compares the image in the window to a reference image with no particles resulting in a difference image. This image data is converted to run-length-code (RLC), thresholded, filtered, and operated on by an algorithm that finds the center of mass of each particle. Before sending these positions to the controller, an algorithm, based on a Kalman filter [37], determines whether each computed position belongs to the same particle or to an unrelated neighboring particle. The 
Kalman filter works by predicting the future position of all particles based on current predicted velocities that are estimated by prior particle position and the time between frames. The filter allows the tracking of individual particles through swarms of other particles.

This image processing and tracking software was coded in C and then compiled into fast assembly routines for the camera. The method finds the position of all the particles in the field of view in less than $25 \mathrm{~ms}$ and passes those $(x, y)$ positions to the control algorithm.

\section{Experimental Sequence}

With the devices and vision system as described earlier, we now describe the experimental sequence to achieve particle steering. We first pressed the microchannel PDMS layer on a microscope glass slide or a Pyrex wafer and filled the channels with ethanol to make the channels hydrophilic. A drop of ethanol at one channel entry filled the entire structure. We then filled the reservoirs with deionized water using a pipette (1000 $\mu \mathrm{L}$ adjustable volume, Eppendorf) and allowed the water to mix with the ethanol. The water/ethanol solution wicks from the reservoirs into the chamber to fill the entire device. Ethanol evaporates faster than water and so we placed the device on a hot plate, at $40^{\circ} \mathrm{C}$ for $30 \mathrm{~min}$, to preferentially evaporate the ethanol. Then all reservoirs were once again filled with deionized water to flush the device. This filling procedure was reliable and eliminated air bubbles.

Next we placed the device onto the inverted microscope and positioned it with the $x-y$ stage to center the control chamber into the camera image plain. We inserted platinum electrodes into the reservoirs by hand and introduced particles into the system through one of the eight reservoirs. A voltage was applied on an opposing electrode to create an electroosmotic flow that moved the particles into the control region.

Before carrying out a particle steering experiment, we need to find the net mobility of the particles (electroosmotic plus electrophoretic), and provide that number to the model (Section V-A), so that the multiparticle steering algorithm (Section $\mathrm{V}-\mathrm{C}$ ), which operates on the model, has an approximately correct net mobility parameter. We measure the net particle mobility $(m=c+u)$ on-line by applying a constant $10 \mathrm{~V}$ actuation at one electrode, while all the other electrodes are set to zero, and measuring the resulting velocity of one particle in the straight channel leading away from the activated electrode. Measurements for the bead and cell velocities yield net mobilities $m=-16$ to $-35 \times 10^{-9} \mathrm{~m}^{2} \mathrm{~V}^{-1} \mathrm{~s}^{-1}$ for the $5.0 \mu \mathrm{m}$ beads, $m=-32$ to $-60 \times 10^{-9} \mathrm{~m}^{2} \mathrm{~V}^{-1} \mathrm{~s}^{-1}$ for the $2.2 \mu \mathrm{m}$ beads, and $m=-20$ to $-40 \times 10^{-9} \mathrm{~m}^{2} \mathrm{~V}^{-1} \mathrm{~s}^{-1}$ for the yeast cells. The more uncertain on-line measurements largely overlap the off-line measurements (see Section IV-B) for the 5.0- $\mu \mathrm{m}$ beads $(-20.8 \pm 2.0)$ and the $2.2-\mu \mathrm{m}$ beads $(-55.5 \pm 12.0)$, but the two measurement techniques provide different results for the yeast cells. The results from the on-line measurements are used in the control algorithm because they provide a measure of the mobility of the particles in the control chamber, as opposed to mobilities of particles in a different device. All the experimental results of Section VI-B were achieved using mobility data from the on-line measurements.
To carry out the particle steering control, we choose (by labeling particles within the vision algorithm by user directed mouse clicks) particles of interest from the numerous particles floating in the control region, and we assign desired paths to these chosen particles. The vision system tracks each of these particles individually, and the control algorithm creates spatially and time-varying flow fields that steers all these chosen particles along their desired paths. The vision system images and the control electrode voltages are updated every 0.20 and $0.033 \mathrm{~s}$, for the single and multiparticle steering experiments, respectively (in the older, single particle setup the camera and software were not yet synchronized).

\section{PArticle SteEring Control Algorithms}

In this section we describe a model of the fluid flow and particle motion in our devices, then we discuss a simple control algorithm used to steer a single particle, and a more sophisticated control algorithm that we use to independently steer multiple particles at once.

\section{A. Model of Fluid and Particle Motion}

In order to create the control algorithm that steers multiple particles independently, we require a model that describes the (neutral or charged) particle motions that results from any electrode actuation. It is possible to design a control algorithm for single particle steering without reference to a model but, even in that case, a model provides valuable insight. Details of our model are covered in [16] and are summarized next.

Fluid dynamics in our devices is described by the Navier Stokes equations [38]. Electric fields are governed by Laplace's equation, the electrostatic limit of Maxwell's equations [39]. Electroosmotic slip velocity boundary conditions [24] are enforced at the floor and ceiling of the devices. The vertical dimension is assumed small compared to the planar device dimensions. We also assume that the voltage is varied smoothly and slowly. The result is that the fluid flow simplifies to a uniform velocity profile in the vertical direction, and its velocity field at any time and place is given by a coefficient times the electric field, i.e., $\vec{V}=u \vec{E}$ [16] where $u$ is the electroosmotic mobility.

If the particles are neutral and sufficiently small they flow along with the fluid. This electroosmotic velocity of the particles $\vec{V}_{E O}$ is given by $\vec{V}_{E O}=u \vec{E}$. If the particles are charged then they also experience electrophoretic velocities $\vec{V}_{E P}$ given by $\vec{V}_{E P}=$ $c \vec{E}$ [24], [25], where $c$ is the electrophoretic mobility. Net particle velocity is therefore $\vec{V}_{\text {Net }}=\vec{V}_{\mathrm{EO}}+\vec{V}_{\mathrm{EP}}=(u+c) \vec{E}=m \vec{E}$ where $m$ is the net particle mobility. Variations in the electroosmotic zeta potential and the amount of charge on the particles can change the coefficient $m$, but the control algorithm is robust to these variations. The net particle mobility coefficient $m$ is measured before we begin our steering experiments (see Section IV-D). Particles also exhibit Brownian motion, which is treated by an appropriate noise term in the model [16].

\section{B. Single Particle Steering Control Algorithm}

Even a very simple control algorithm is sufficient to steer a single particle. The basic feedback control steering concept is described in Section II. In the four-electrode device, the north and south electrodes can create a primarily north or south flow 


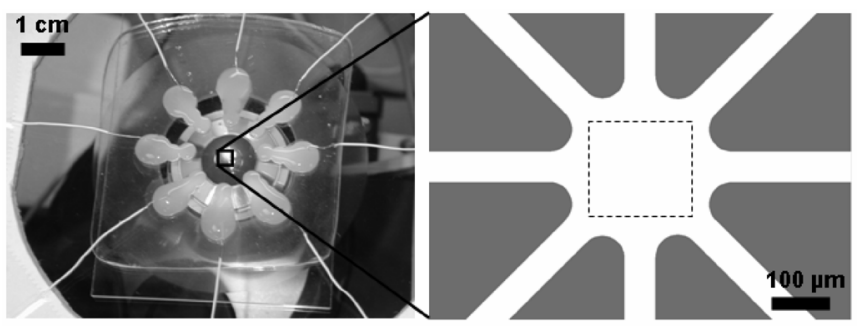

Fig. 4. Left: the 8-electrode PDMS on glass device. Here the white bulb shapes are the eight reservoirs (big reservoirs are used to minimize surface tension driven pressure flows and electrochemical effects), platinum wire electrodes are brought in contact with the fluid in the reservoirs. In these wells, the 8 channels (each $7 \mathrm{~mm}$ long, $50 \mu \mathrm{m}$ wide, $11 \mu \mathrm{m}$ deep) are not visible, and a blue LED light (used to illuminate the fluorescent particles) brightly illuminates the center of the device. Right: a mask (a zoom) of the particle steering region (300 $\mu \mathrm{m}$ diameter, $11 \mu \mathrm{m}$ deep).

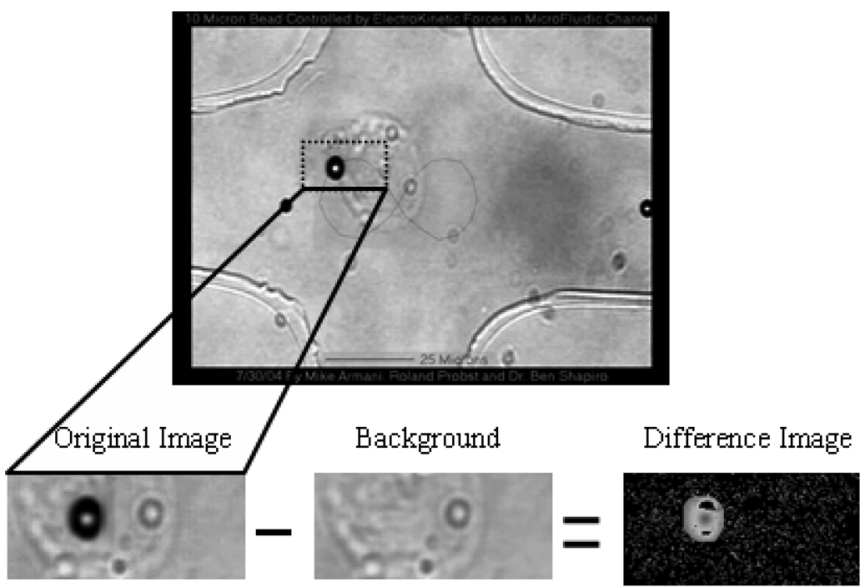

Threshold

RLC

Filter

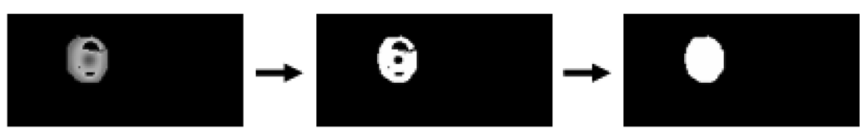

Finde Object

Center of Mass
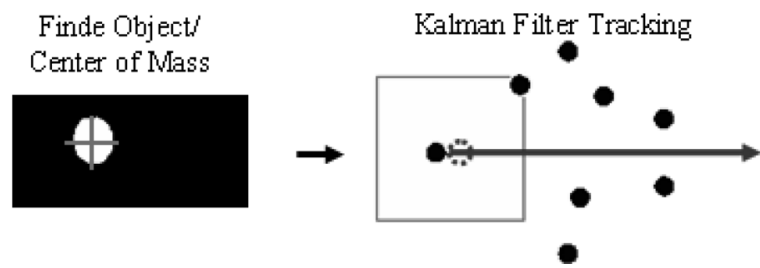

Fig. 5. The real time algorithm for finding the $(x, y)$ positions of all the particles. A reference image is taken of the device when there are no particles in the chamber. Then, for each incoming camera image, we subtract away the reference image to create a differential image that isolates the pixels corresponding to the moving particles. The differential image is threshold to remove the effects of noise and the centroid for each particle is computed. A Kalman filter allows tracking of individual particles.

and the east and west electrodes can create an east or west flow. In the simplest control algorithm [15], each electrode pair corrects for particle position along its own axis independently of the other electrode pair.

Specifically, if the particle is more then five pixels north from its desired north/south elevation then the north electrode applies $+30 \mathrm{~V}$ and the south electrode applies $-30 \mathrm{~V}$. Once the particle is within five camera pixels, a lower $\pm 12 \mathrm{~V}$ actuation is used. The east/west electrodes work the same way. This algorithm

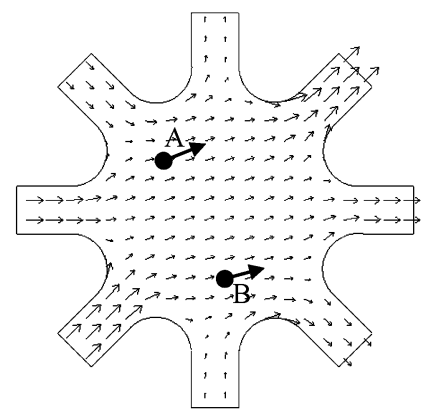

fluid mode 1

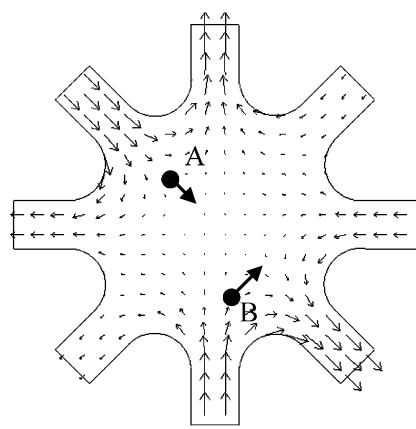

fluid mode 5

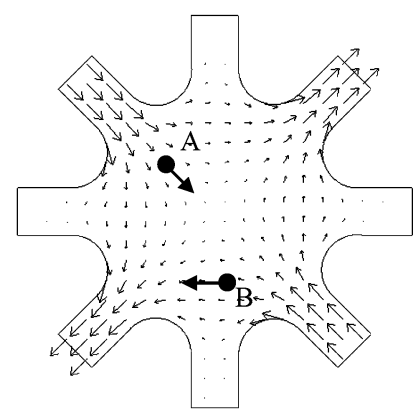

fluid mode 3

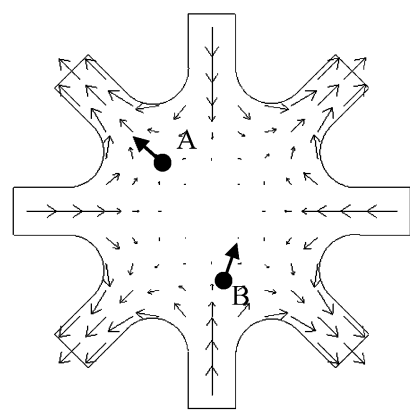

fluid mode 7
Fig. 6. Electroosmotic microflow modes for the 8-electrode device of Fig. 4. The first, third, fifth, and seventh electroosmotic fluid modes are shown, as computed by the fluid flow model of Section V-A and [16]. The two neutral particles $\mathrm{A}$ and $\mathrm{B}$ (shown as black dots above) will then experience the velocities shown by the arrows. To actuate mode 5 , the following voltages must be applied at the eight electrodes: $-4.7 \mathrm{~V}, 1.2 \mathrm{~V}, 2.67 \mathrm{~V},-4.36 \mathrm{~V}, 4.8 \mathrm{~V},-1.15 \mathrm{~V},-2.75 \mathrm{~V}, 4.27$ $\mathrm{V}$ (listed clockwise from the north electrode).

is sufficient to keep a yeast cell within one pixel of its desired trajectory (see Fig. 9). For our current optical setup, this pixel error corresponds to a tracking accuracy of $1 \mu \mathrm{m}$.

\section{Multiple Particle Steering Control Algorithm}

The multiparticle steering control algorithm is more sophisticated than the single particle algorithm: its operation relies on inversion of the flow and electric fields predicted by the model of Section V-A. The algorithm is described in detail in [16] and is explained briefly below. Although the algorithm reported in [16] was validated only against numerical simulations, it turns out that we did not have to change it to implement particle steering in our eight electrode experiments. We also note that the square device geometry described in [16] differs from the current (more practical) central hub with channels and reservoirs geometry, but the control algorithm operation is the same for both geometries.

The eight-electrode device shown in Fig. 4 can create seven independent electric/fluid modes (one of the eight electrodes acts as ground so only 7 degrees of freedom remain). Four of these seven modes are shown in Fig. 6. The key point is that the different modes force particles at different locations in different directions (see particles A and B in Fig. 6): by intelligently actuating a combination of modes, we can force all the particles towards the right locations at each instant in time. Since each particle has two degrees of freedom (an $\mathrm{x}$ and a $\mathrm{y}$ position), the eight-electrode device can precisely control up to three particles 


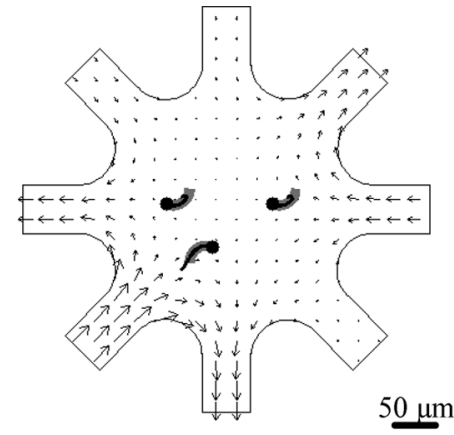

time $=10 \mathrm{~s}$

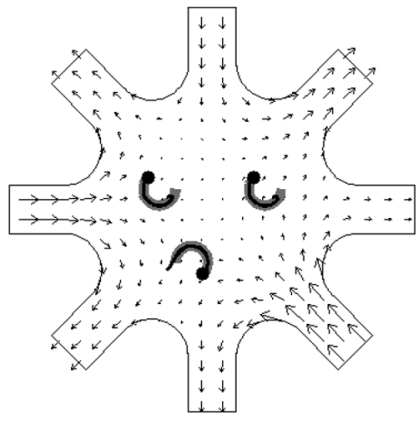

time $=20 \mathrm{~s}$

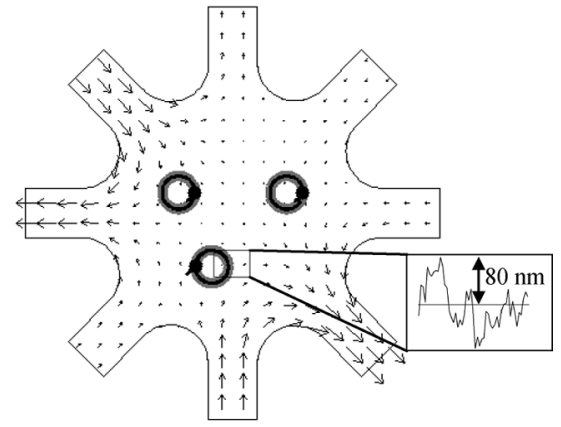

time $=30 \mathrm{~s}$

Fig. 7. Steering of three charged particles ( $2.5 \mu \mathrm{m}$ diameter, electrophoretic mobility $\left.c=-10 \times 10^{-9} \mathrm{~m}^{2} \mathrm{~V}^{-1} \mathrm{~s}^{-1}\right)$ by electroosmotic fluid actuation and feedback control in the 8-electrode device (in simulation). The view is from above. Three time instants are shown. The vectors are the resulting fluid velocities in the device, the small black dots are the three particles to be controlled, the thick grey curves indicate the desired trajectories of the particles, and the thin black curves indicate the actual trajectories of the particles. In this simulation, the particles start from initial positions that do not correspond to their initial desired positions, and the system has a realistic amount of Brownian thermal noise corresponding to particles of this size, at room temperature, in water.

(particle degrees of freedom $3 \times 2=6 \leq 7$ actuation degrees of freedom).

The control algorithm works in two parts. The first part (inversion) determines the electrode voltages that would steer the particles along desired directions if there were no errors or sources of noise. The second part compares the actual particle locations to the desired locations and applies voltages to correct for any particle position errors (feedback). In both parts, the necessary electrode actuation is predicted based on the model of Section V-A. The flow or electric field actually created in the device will differ from the one predicted by the model, but so long as the flow field is sufficiently accurate to move the particles from where they are to closer to where they should be, the scheme will work-even an imperfect model allows effective feedback control [17], [40].

The first (inversion) portion of the control computes the electrode voltages that will create the desired particle velocities. Based on the model of Section V-A, there is a linear mapping between the applied electrode voltages and the resulting velocities of the particles. The mapping is linear because the particles follow the electric field, either due to electroosmosis (neutral particles) or electroosmosis and electrophoresis (charged particles), and the electric field is linear in the applied actuator voltages. This linear mapping is different for different particle locations. At each instant in time, for the current set of particle positions, we compare the linear subspace of achievable particle velocities with a vector of all the desired particle velocities. We then solve a least squares problem to find the electrode voltages that will create a set of achievable velocities (in the achievable velocities subspace) that is as close as possible to the vector of desired particle velocities. (If we have more actuators than particle degrees of freedom then we can solve this problem exactly. If not, the least squares solution provides the best possible answer.) The process repeats at each time instant for each new set of particle positions.

The second (feedback) portion of the controller compares the actual positions of the particles at each instant to their desired locations. The error between the desired and actual positions is multiplied by a nonlinear feedback gain (feedback linearization [41]) to create a set of correction electrode voltages that are added to the voltages computed in the inversion step. The benefit of the feedback linearization approach is that it guarantees global stability [41]: even if the position of the particles deviates by an arbitrary amount from their desired trajectories, the feedback control algorithm will still force the particles back to their desired paths (see Fig. 13). Fig. 7 shows three particles being steered using the above algorithm in a simulation of the 8-electrode device.

\section{EXPERIMENTAL RESULTS}

Here we show experimental results for steering of single and multiple, charged and nearly neutral, particles along various desired trajectories.

\section{A. Steering a Single Particle}

Fig. 8 shows the steering of a charged bead along a Fig. 8 in the 4-electrode device. The surface charge on the bead leads to an approximate electrophoretic mobility of $(-57.3 \pm 5.6) \times$ $10^{-9} \mathrm{~m}^{2} \mathrm{~V}^{-1} \mathrm{~s}^{-1}$. The precise surface charge on the bead is not known (it depends in a complicated way on the $\mathrm{pH}$, temperature, concentration and type of impurities in the surrounding medium), and is not required by the control algorithm.

The experiment of Fig. 8 was performed before we had optimized the 4-electrode single particle steering device, as a result the particle steering accuracy is poor. For the field of view used in the single particle experiments, each pixel in the camera corresponds to a distance of $917 \mathrm{~nm}$ in the $x$ direction and $687 \mathrm{~nm}$ in the $y$ direction. The deviation between the actual and desired path in Fig. 8 is about 3 pixels, hence, our steering accuracy here is about $3 \mu \mathrm{m}$.

Fig. 9 shows the steering of a $5-\mu$ m-diameter yeast cell along a University of Maryland (UMD) path. Yeast cell electrophoretic mobilities have been reported to vary between $(-11$ to -21$) \times 10^{-9} \mathrm{~m}^{-2} \mathrm{~V}^{-1} \mathrm{~s}^{-1}$ [33]. The yeast cell is less charged than the polystyrene bead but it is still not perfectly neutral. Again, the exact charge or mobility of the cell is not important in terms of control and here the chosen cell was steered to an accuracy of one camera pixel (better than $1 \mu \mathrm{m}$ ) without using precise charge or mobility information. This experiment was an optimized version of the one in Fig. 8. 


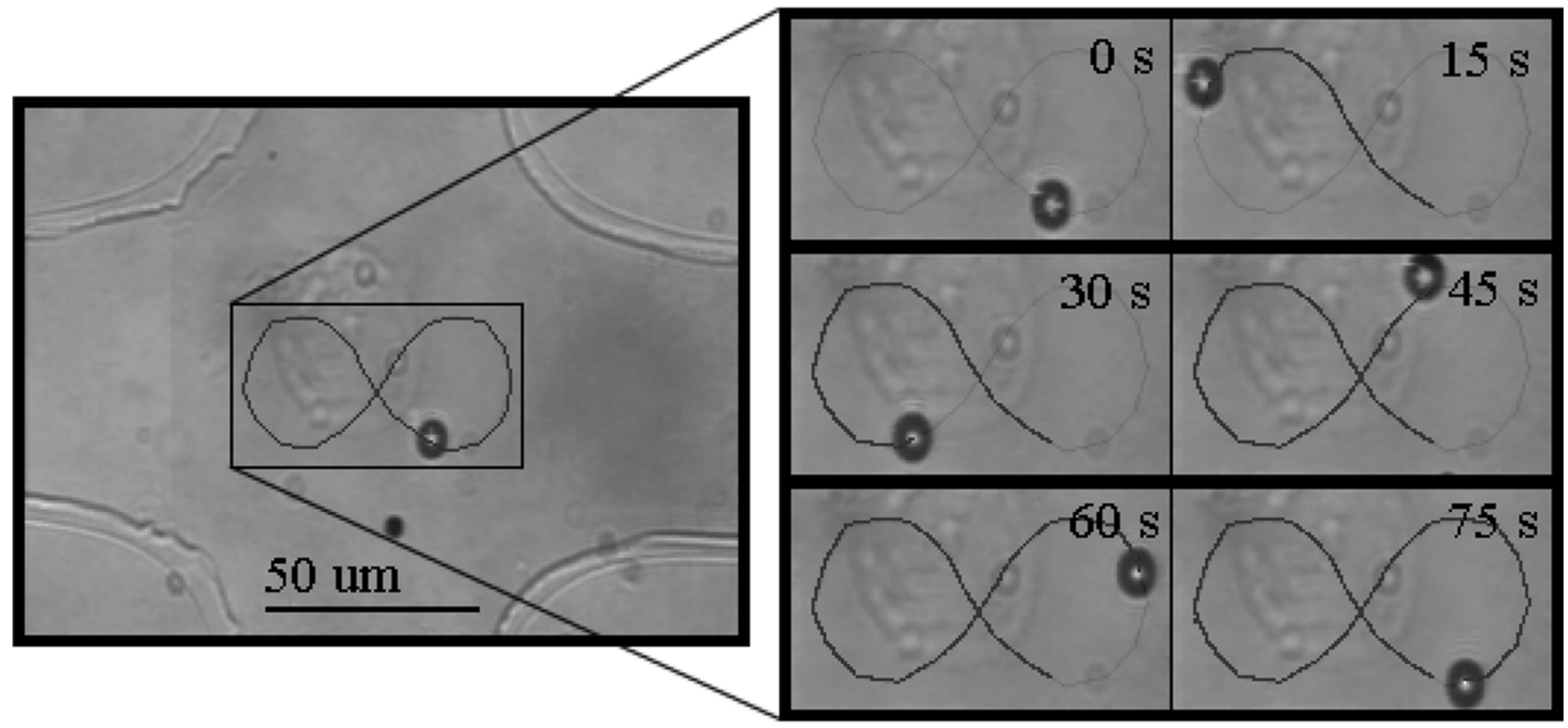

Fig. 8. Control of a bead with significant surface charge along figure " 8 ". The bead has an approximate electrophoretic mobility of $c=(-57.3 \pm 5.6) \times$ $10^{-9} \mathrm{~m}^{2} \mathrm{~V}^{-1} \mathrm{~s}^{-1}$. (By comparison, the electroosmotic mobility of our PDMS devices is $u=(36.5 \pm 3.6) \times 10^{-9} \mathrm{~m}^{2} \mathrm{~V}^{-1} \mathrm{~s}^{-1}$.) Left: photograph of the microfluidic devices with the figure " 8 " path superimposed on the image. Right: the actual path of the chosen $5 \mu$ m polystyrene bead (Polysciences Inc.) (black circle) in the feedback control experiment. Snapshots are shown at six equally spaced times. The bead follows the required trajectory to within $3 \mu \mathrm{m}$.

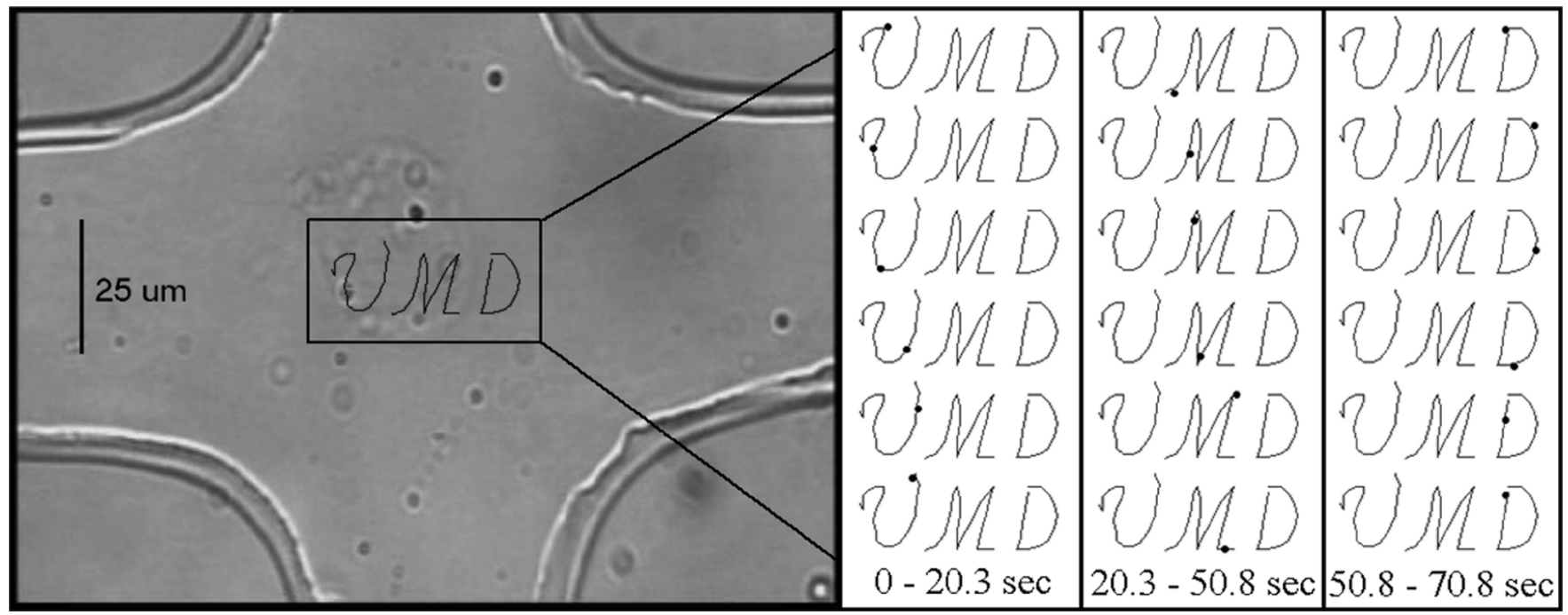

Fig. 9. Steering of a slightly charged yeast cell along a UMD path. The cell has an approximate electrophoretic mobility of $c=(-23.3 \pm 6.9) \times 10^{-9} \mathrm{~m}^{2} \mathrm{~V}^{-1} \mathrm{~s}^{-1}$ Left: photograph of the microfluidic devices with the cursive "UMD" path overlaid on the image. Right: the actual path of the chosen 5- $\mu \mathrm{m}$ yeast cell (Red Star yeast) (black dot) in the feedback control experiment. Snapshots are shown at 6 equally spaced times for each letter. The yeast cell follows the required trajectory to within $1 \mu \mathrm{m}$. (This experiment was an optimized version of the Fig. 8 experiment.)

\section{B. Steering Multiple Particles}

Our 8-electrode device has 7 degrees of freedom (one electrode is ground) and can precisely steer up to three particles (each particle has 2 degrees of freedom). Fig. 10 shows the simultaneous steering of three polystyrene beads along three circular paths.

As noted in Section $\mathrm{V}$, the control algorithm can trap particles by forcing a particle to move back to its desired position whenever it deviates away due to external forces. This can be done even while other particles are being steered along their paths.
Fig. 11 shows the steering of two beads along two circular paths while a third bead is controlled to stay at a fixed location. The better than $1 \mu \mathrm{m}$ trapping accuracy is set by the optical resolution of the vision system.

Both neutral and charged particles can be steered. We did not have access to particles that remain perfectly neutral when immersed in water, but Fig. 12 displays the motion of three yeast cells, which acquire less surface charge than the beads, being steered along two circles and a "UMD" path.

It was noted in Section V-C that the steering control algorithm can correct for large errors, it can steer chosen particles 

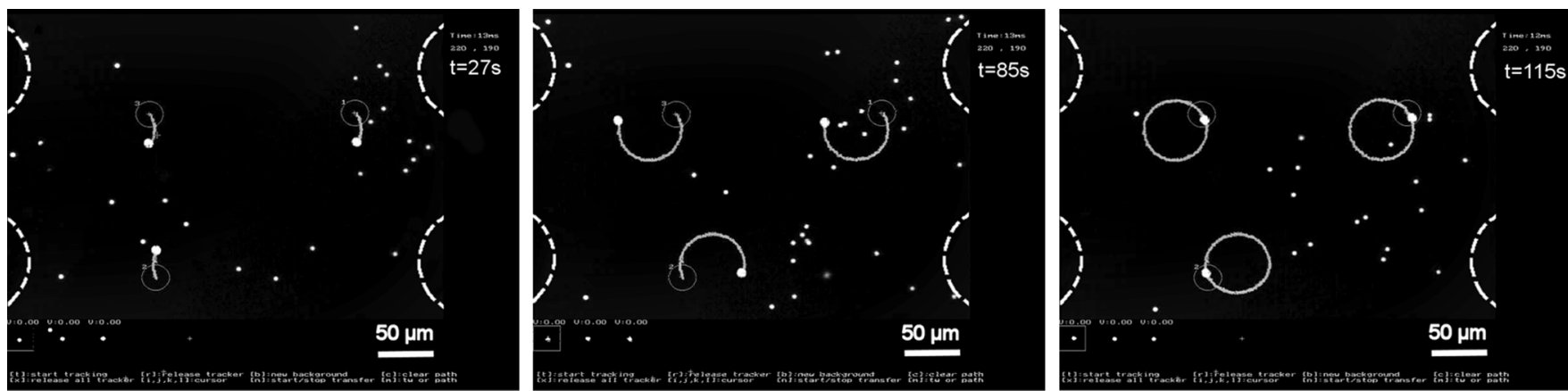

Fig. 10. Steering of three fluorescent beads $(2.2 \mu \mathrm{m}$ diameter, Duke Scientific) with large surface charge (electrophoretic mobility of $-92.0 \pm 15.6 \times$ $\left.10^{-9} \mathrm{~m}^{2} \mathrm{~V}^{-1} \mathrm{~s}^{-1}\right)$ around 3 circles. The electroosmotic mobility of our PDMS devices is $u=(36.5 \pm 3.6) \times 10^{-9} \mathrm{~m}^{2} \mathrm{~V}^{-1} \mathrm{~s}^{-1}$. In the experiment, the fluorescent beads appear as small green dots on a black background and the device geometry, which does not fluoresce, is not visible. Here, the white dots are the beads (enlarged), the blue curves are the actual trajectories that the target beads have traced out (overlaid), and the dashed white curves (also overlaid) show the geometry of the channels and the particle control chamber. Snapshots are shown at three time steps. The three beads are being steered to within an accuracy of one pixel (corresponding to less than $1 \mu \mathrm{m}$ ). The desired paths are not shown because, at this image resolution, they would perfectly underlay the actual paths.
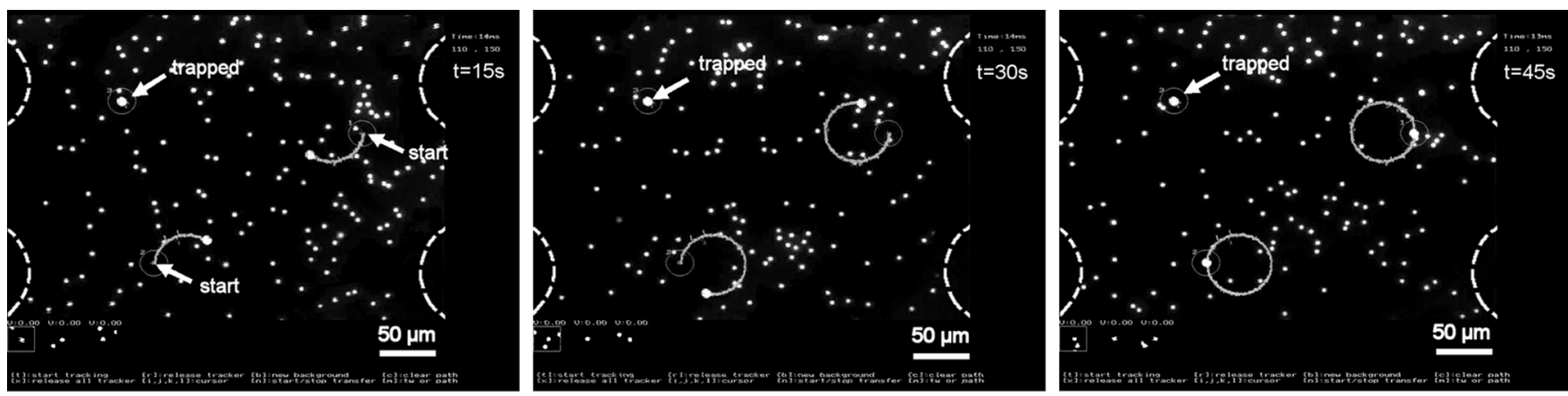

Fig. 11. Steering of 2 fluorescent beads ( $2.2 \mu \mathrm{m}$ diameter, Duke Scientific) around 2 circles while a third bead is held stationary. The trapped bead is marked by an arrow, and is trapped by the control algorithm to an accuracy of better than $1 \mu \mathrm{m}$. Every time the bead deviates from its desired position, a flow is created that pushes the bead back towards its desired location. (The format of the figure is the same as in Fig. 10.)
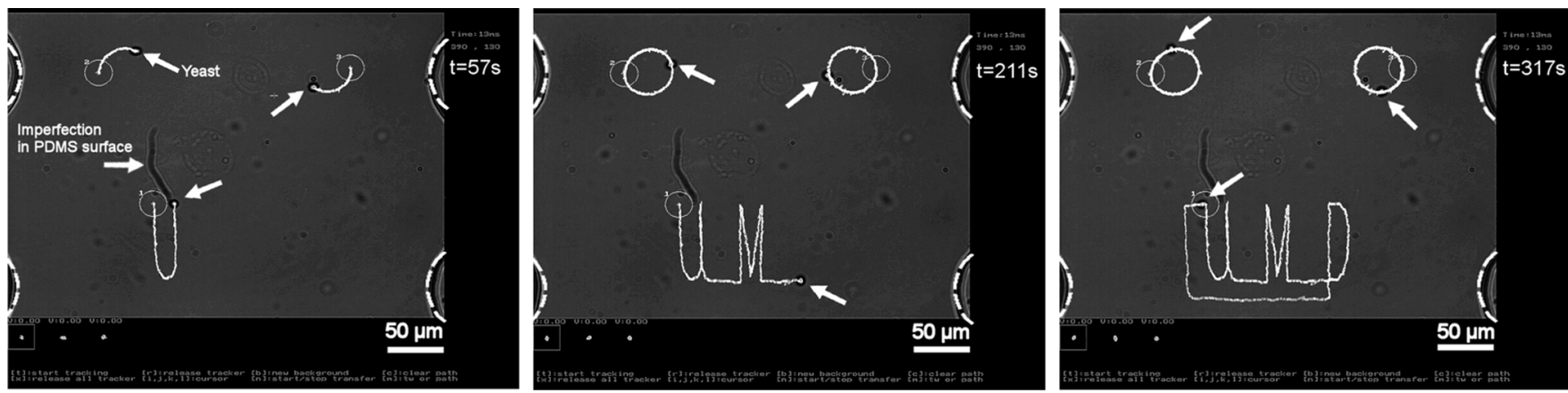

Fig. 12. Steering of three yeast cells $(5 \mu \mathrm{m}$ diameter, Red Star, Giant Food) with small surface charge (electrophoretic mobility $c=(-23.3 \pm 6.9) \times$ $10^{-9} \mathrm{~m}^{2} \mathrm{~V}^{-1} \mathrm{~s}^{-1}$ ) around two circles and a "UMD" path. The cells do not fluoresce. In these images there is no high-pass filter before the camera and the raw images are shown. The yeast cells are visible as small black dots with a white center (the three target cells are marked with a white arrow in each image), and the white curves are the trajectories that the target cells have traced out. The three beads are being steered to within an accuracy of one pixel (corresponding to less than $1 \mu \mathrm{m})$.

to their desired locations even if they are initially far away. This capability is demonstrated in Fig. 13. Here the control has been turned off for $11 \mathrm{~s}$ until the beads have drifted away a large distance, the control is then turned back on and the algorithm drives the original three beads back to their desired paths.

\section{Particle Steering Accuracy}

The spatial accuracy of our particle steering is determined primarily by the field of view associated with a single camera pixel. In the single particle experiments, one camera pixel corresponded to a spatial displacement of $917 \mathrm{~nm}$ in the $x$ direction and $687 \mathrm{~nm}$ in the $y$ direction (the pixels are rectangular). In the multiparticle experiments, a slightly smaller field of view was used, and one pixel corresponded to 625 and $468 \mathrm{~nm}$ distances, respectively. Both the single and multiple particle algorithms steer the particles to within one pixel of their desired paths. This single pixel deviation corresponds to a maximum spatial error of 0.917 and $0.625 \mu \mathrm{m}$ for the single and multiparticle steering experiments, respectively. 


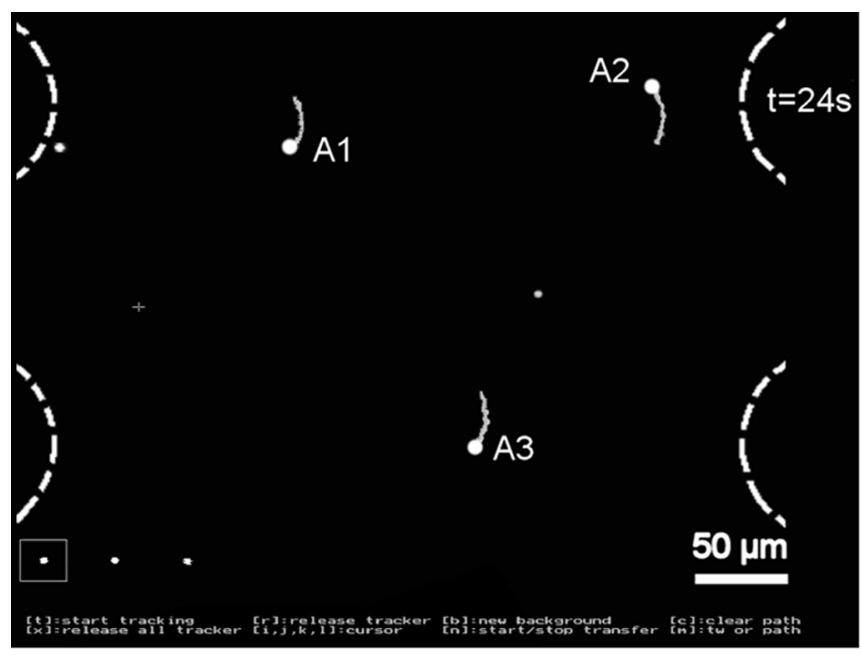

(a)

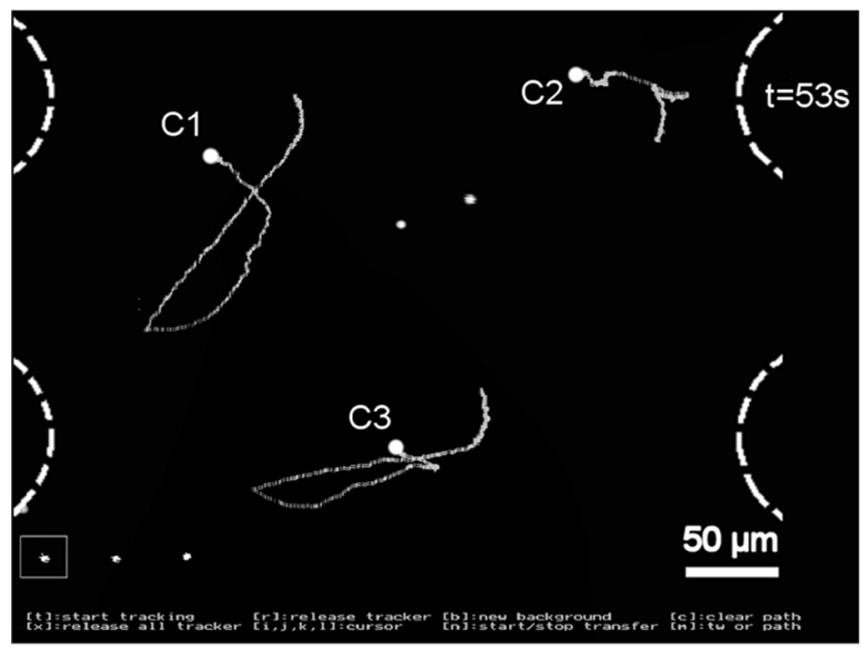

(c)

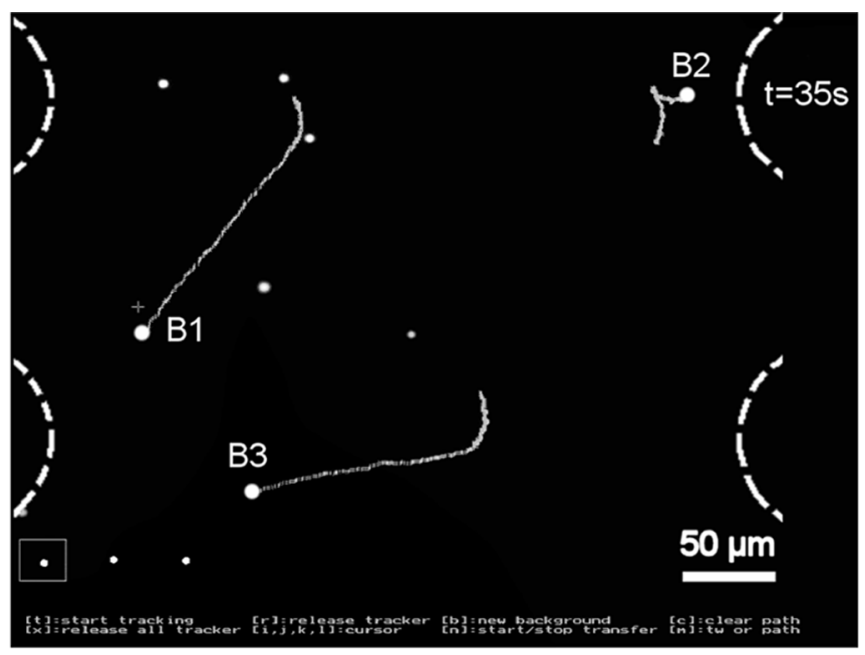

(b)

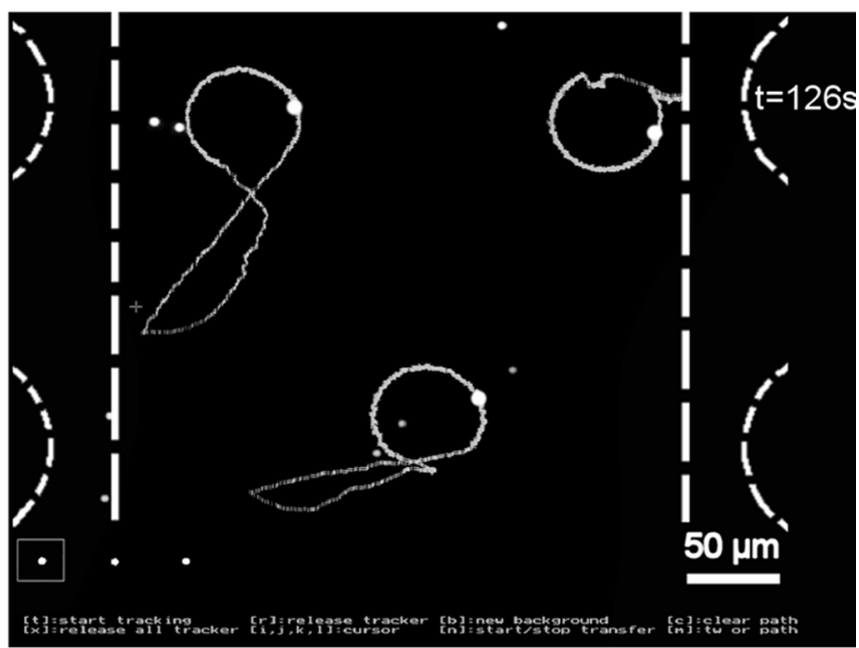

(d)

Fig. 13. The control algorithm can correct for large errors in particle positions. This figure shows steering of three fluorescent beads $(2.2 \mu \mathrm{m}$ diameter, Duke Scientific) around three circles. At time $t=24 \mathrm{~s}$, corresponding to bead positions marked $\mathrm{A}_{1}, \mathrm{~A}_{2}$, and $\mathrm{A}_{3}$, the control was turned off for $11 \mathrm{~s}$, allowing the particles to drift away (primarily due to the slow parasitic flow caused by surface tension forces at the reservoirs) by up to $150 \mu \mathrm{m}$. The control was then turned back on at $\mathrm{t}=35 \mathrm{~s}\left(\mathrm{~B}_{1}, \mathrm{~B}_{2}\right.$, and $\left.\mathrm{B}_{3}\right)$, and the control algorithm steered the three original beads back to their desired positions $\left(\mathrm{C}_{1}, \mathrm{C}_{2}\right.$, and $\left.\mathrm{C}_{3}\right)$. Four time instants are shown. (a) Right before control is turned off. (b) Right before control is turned back on (the three beads have drifted away a large distance). (c) At a time when the beads are back on track. (d) Final time when the beads have completed the remainder of their three circular paths (again to an accuracy of better than $1 \mu \mathrm{m}$ ). The two straight lines in the last image illustrate the left and right boundaries of the control region. In Section V-C, the control voltages scale with the size of the position errors. Position errors are very large and this would lead to very large control voltages. Therefore, the control scheme has been slightly modified: the control gain per particle is scaled in such a way that the voltage remains within the allowable $[-10,+10] \mathrm{V}$ range. (Format of the figure is the same as in Fig. 10.)

The other sources of errors are much smaller. There is an error due to the finite control update time. Control inputs are updated every $0.20 \mathrm{~s}$ (for the older single particle experiments) and every $0.033 \mathrm{~s}$ (for the multiparticle experiments). During this control update time $d t$ the particle can deviate away from its desired location due to Brownian motion. The deviation amount $\delta_{\mathrm{B}}$ is predicted by [24]

$$
\begin{aligned}
\delta_{B} & =\sqrt{\frac{k T d t}{3 \pi \eta a}} \\
& =\sqrt{\frac{1.3807 \times 10^{-23} \mathrm{JK}^{-1} \times 300 \mathrm{~K} \times 0.033 \mathrm{~s}}{3 \times 3.14 \times 0.001 \mathrm{Ns} / \mathrm{m}^{2} \times 2.5 \times 10^{-6} \mathrm{~m}}} \\
& =76 \mathrm{~nm}
\end{aligned}
$$

where $k$ is the Boltzman constant, $T$ is the ambient temperature, $d t$ is the time interval, $\eta$ is the dynamic viscosity of water, and $a$ is the particle radius. During this control update time, there is also an error created because an old control actuation is being applied to a slightly new particle position. This creates a position error $\delta_{\mathrm{C}}$ that is bounded by the maximum particle velocity $\left(\mathrm{V}<5 \mu \mathrm{m} \mathrm{s}^{-1}\right)$ times the update control time interval $d t$, the resulting error $\delta_{C} \leq V \times d t$ is less than $0.25 \mu \mathrm{m}$.

To close, we compare the attributes of laser tweezers and our feedback flow control method in Table I.

\section{CONCLUSION}

Feedback control allows simple microfluidic devices to execute sophisticated tasks. We demonstrate experimentally that vi- 
TABLE I

COMPARISON OF ATtRibutes of PARTICle STEERING By LASER TwEEZERS AND FEEDBACK Flow CONTROL

\begin{tabular}{|l|l|l|l|l|l|l|l|l|}
\hline & $\begin{array}{l}\text { Number of } \\
\text { particles }\end{array}$ & $\begin{array}{l}\text { Positioning } \\
\text { accuracy }\end{array}$ & $\begin{array}{l}\text { Applied } \\
\text { forces }\end{array}$ & $\begin{array}{l}\text { Steer in } \\
2 \text { or 3D }\end{array}$ & $\begin{array}{l}\text { Type particles } \\
\text { steered }\end{array}$ & $\begin{array}{l}\text { Cost of } \\
\text { system }\end{array}$ & $\begin{array}{l}\text { Hand- } \\
\text { held }\end{array}$ & $\begin{array}{l}\text { Years under } \\
\text { development }\end{array}$ \\
\hline Laser & up to 400 & $<10 \mathrm{~nm}$ & $100 \mathrm{aN}-$ & $3 \mathrm{D}$ & neutral, dielectric, & $\$ 10 \mathrm{k}$ & no & 30 \\
$100 \mathrm{pN}$ & & $10 \mathrm{~nm}-10 \mu \mathrm{m}$ size & $-400 \mathrm{k}$ & & \\
\hline Flow Control & 3 using 8 & $<1 \mu \mathrm{m}$ & $\sim 20 \mathrm{pN}$ & $2 \mathrm{D}$ & any visible & $<\$ 5 \mathrm{k}$ & possible & 2 \\
& & & & & & & & \\
\hline
\end{tabular}

Attributes for laser tweezers taken from [2], [14]. To be steered by a laser tweezer, the dielectric constant of the particle must differ from the surrounding medium. Our applied forces, for neutral particles, have been estimated using the classical Stokes drag law $\mathrm{F}=-6 \pi \eta \mathrm{r} \mathrm{V}$ [24] where $\eta$ is the dynamic viscosity of water at room temperature, $r$ is the radius of the particles, and $V$ is the velocity of the flow created in our devices. These applied force have not been maximized and are low because the $\mathrm{V} \leq 100 \mu \mathrm{m} / \mathrm{s}$ fluid velocities are slow. The cost of the flow control system is set primarily by the cost of the vision system (the microscope and camera). A contact imager chip [43] would allow the system to be miniaturized into a handheld format (the necessary voltage requirements can be provided by a small battery, and the control algorithms can be implemented on a chip). Existing contact imagers have pixels sizes as small as $20 \mu \mathrm{m} \times 20 \mu \mathrm{m}$ and they can implement center-of-gravity algorithms that can locate objects to subpixel resolution [44]. Thus, a contact imager vision system will still allow control of particles to micrometer precision. It will also decrease the cost of the system to that of a PDMS device, a battery, and two chips (one for imaging and one for control).

sion-based feedback control allows a four- and eight-electrode PDMS device to steer single and multiple particles along arbitrary paths with micrometer precision. At each time instant a vision system identifies the current locations of the particles, a control algorithm determines the actuator voltages that will create a fluid flow (for electroosmosis actuation) or an electric field (for electrophoretic actuation) to move the neutral or charged particles from their current locations toward their next desired positions, and the necessary flow or electric field is then created by voltage actuation.

This control permits the steering of any visible particles (neutral particles are carried by the flow, charged particles are also actuated electrophoretically), for neutral particles the method is noninvasive because it does not actuate the particles directly but instead moves them by transporting the surrounding medium, multiple particles can be steered and trapped at the same time, and the imaging system and control algorithm will be integrated on-chip in next-generation devices thus allowing the entire system to be integrated into a handheld format. Our approach is an example of how field inversion (finding the required flow or electric field) plus error correction (feedback) allows a MEMS designer to extract more functionality (here, multiparticle steering) from existing devices. We have shown a similar steering capability (in simulations) for electrowetting devices using flow fields created by electrical surface tension actuation [42] and it should also be possible to steer multiple particles by other fields, such as pressure flows or dielectrophoretic force fields.

\section{ACKNOWLEDGMENT}

The authors would like to thank J. G. Santiago for his helpful comments on interface and colloid chemistry and its relation to charge on device and particle surfaces.

\section{REFERENCES}

[1] A. Ashkin, "History of optical trapping and manipulation of small-neutral particles, atoms, and molecules," IEEE J. Sel. Topics Quantum Electron., vol. 6, no. 6, pp. 841-856, 2000.
[2] J. E. Curtis, B. A. Koss, and D. G. Grier, "Dynamic holographic optical tweezers," Opt. Commun., vol. 207, no. 1-6, pp. 169-175, 2002.

[3] D. G. Grier, "A revolution in optical manipulation," Nature, vol. 424, pp. 810-816, 2003.

[4] P. Y. Chiou and A. T. Ohta et al., "Massively parallel manipulation of single cells and microparticles using optical images," Nature, vol. 436, pp. 370-372, 2005.

[5] M. P. Hughes, "Strategies for dielectrophoretic separation in laboratory-on-a-chip systems," Electrophoresis, vol. 23, pp. 2569-2582, 2002.

[6] T. Muller et al., "Trapping of micrometre and sub-micrometre particles by high-frequency electric fields and hydrodynamic forces," J. Phys. D: Appl. Phys., vol. 29, pp. 340-349, 1996.

[7] R. Pethig and M. S. Talary et al., "Enhancing traveling-wave dielectrophoresis with signal superposition," IEEE Eng. Med. Biol. Mag., vol. 22, pp. 43-50, 2003.

[8] H. Morgan and N. G. Green et al., "Large-area travelling-wave dielectrophoresis particle separator," J. Micromech. Microeng., vol. 7, no. 2, pp. 65-70, 1997.

[9] H. M. Hertz, "Wave acoustic trap for nonintrusive positioning of microparticles," J. Appl. Phys., vol. 78, pp. 4845-4849, 1995.

[10] H. Lee, T. P. Hunt, and R. M. Westervelt, "Magnetic and electric manipulation of a single cell in fluid," Material Research Society Symp. 2004, Mater. Res. Soc.

[11] Y. Fukuta et al., "A MEMS array for pneumatic conveyor and its control based on distributed systems," Micro-Electro-Mechanical Systems (MEMS). Miami, FL, 2005.

[12] A. E. Cohen, "Control of nanoparticles with arbitrary two-dimensional force fields," Phys. Rev. Lett., vol. 94, p. 118102, 2005.

[13] A. E. Cohen and W. E. Moerner, "Method for trapping and manipulating nanoscale objects in solution," Appl. Phys. Lett., vol. 86, 2005.

[14] J. P. Hoogenboom et al., "Patterning surfaces with colloidal particles using optical tweezers," Appl. Phys. Lett., vol. 80, no. 25, pp. 4828-4830, 2002.

[15] M. Armani et al., "Using feedback control and micro-fluidics to steer individual particles," in 18th IEEE Int. Conf. Micro Electro Mechan. Syst., Miami, FL, 2005.

[16] S. Chaudhary and B. Shapiro, "Arbitrary steering of multiple particles at once in an electroosmotically driven microfluidic system," IEEE Trans. Contr. Syst. Technol., vol. 14, no. 4, pp. 669-680, Jul. 2006.

[17] J. C. Doyle, B. A. Francis, and A. R. Tannenbaum, Feedback Control Theory. New York: Macmillan, 1992.

[18] R. M. Murray et al., Control in an Information Rich World. : Air Force Office of Scientific Research (AFOSR), 2002.

[19] Y. N. Xia and G. M. Whitesides, "Soft lithography," Ann. Rev. Mater. Sci., vol. 28, pp. 153-184.

[20] D. C. Duffy et al., "Rapid prototyping of microfluidic systems in poly(dimethylsiloxane)," Anal. Chem., vol. 70, no. 23, pp. 4974-4984, 1998. 
[21] G. M. Whitesides and J. C. McDonald et al., "Fabrication of microfluidic systems in poly(dimethylsiloxane)," Electrophoresis, vol. 21, no. 1, pp. 27-40, 2000.

[22] Y. B. Acar, A. N. Alshawabkeh, and R. A. Parker, Theoretical and Experimental Modeling of Multi-Species Transport in Soils Under Electric Fields Project Summary. Cincinnati, OH: U.S. Environmental Protection Agency, National Risk Manage. Res. Lab., 1997.

[23] T. S. Light, E. A. Kingman, and A. C. Bevilacqua, "The conductivity of low concentrations of $\mathrm{CO} 2$ dissolved in ultrapure water from 0-100 ${ }^{\circ}$ C," in 209th Amer. Chem. Soc. Nat. Meet., Anaheim, CA, 1995.

[24] R. F. Probstein, Physicochemical Hydrodynamics: An Introduction, 2nd ed. New York: Wiley, 1994.

[25] P. C. Hiemenz and R. Rajagopalan, Principles of Colloid and Surface Chemistry, 3rd ed. New York, Basel, Hong Kong: Marcel Dekker, 1997.

[26] Y. Liu and J. C. Fanguy et al., "Dynamic coating using polyelectrolyte multilayers for chemical control of electroosmotic flow in capillary electrophoresis microchips," Anal. Chem., vol. 72, pp. 5939-5944, 2000.

[27] A. M. Spehar et al., "Electrokinetic characterization of poly(dimethylsiloxane) microchannels," Electrophoresis, vol. 24, no. 21, pp. 2674-2678, 2003.

[28] N. G. Vanifatova et al., "Separation of unmodified polystyrene nanosphere standards by capillary zone electrophoresis," J. Chromatogr. A., vol. 898, no. 2, pp. 257-263, 2000.

[29] H. K. Jones and N. E. Ballou, "Separations of chemically different particles by capillary electrophoresis," Anal. Chem., vol. 62, no. 22, pp. 2484-2490, 1990

[30] L. Kremser, D. Blaas, and E. Kenndler, "Capillary electrophoresis of biological particles: viruses, bacteria, and eukaryotic cells," Electrophoresis, vol. 25, no. 14, pp. 2282-2291, 2004.

[31] D. A. Lytle et al., "Electrophoretic mobilities of Escherichia coli O157:H7 and wild-type Escherichia coli strains," Appl. Environ. Microbiol., vol. 65, no. 7, pp. 3222-3225, 1999.

[32] J. R. Glynn et al., "Capillary electrophoresis measurements of electrophoretic mobility for colloidal particles of biological interest," Appl. Environ. Microbiol., vol. 64, no. 7, pp. 2572-2577, 1998.

[33] T. Nakari-Setala et al., "Expression of a fungal hydrophobin in the Saccharomyces cerevisiae cell wall: effect on cell surface properties and immobilization," Appl. Environ. Microbiol., vol. 68, no. 7, pp. 3385-3391, Jul. 1, 2002.

[34] F. F. Vargas et al., "Surface charge of endothelial cells estimated from electrophoretic mobility," Membrane Biochem., vol. 8, no. 4, pp. 221-227, 1989.

[35] B. A. Smith, B. R. Ware, and R. S. Weiner, "Electrophoretic distributions of human peripheral blood mononuclear white cells from normal subjects and from patients with acute lymphocytic leukemia," Proc Natl Acad. Sci. USA, vol. 73, no. 7, pp. 2388-2391, 1976.

[36] Elimelech, "Effect of electrolyte type on the electrophoretic mobility of polystyrene latex colloids," Colloids Surf., vol. 44, pp. 165-178, 1990, M.a.O.M.C.R

[37] R. Kalman and Emil, "A new approach to linear filtering and prediction problems," Trans. ASME-J. Basic Eng., ser. D, vol. 82, pp. 35-45, 1960.

[38] R. L. Panton, Incompressible Flow, 2nd ed. New York: Wiley, 1996.

[39] R. P. Feynman, R. B. Leighton, and M. Sands, The Feynman Lectures on Physics. Reading, MA: Addison-Wesley, 1964.

[40] K. Zhou, J. C. Doyle, and K. Glover, Robust and Optimal Control. Englewood Cliffs, NJ: Prentice-Hall, 1996.

[41] H. K. Khalil, Nonlinear Systems. Englewood Cliffs, NJ: PrenticeHall, 2002.

[42] S. Walker and B. Shapiro, "A control method for steering individual particles inside liquid droplets actuated by electrowetting," Lab on a Chip J., vol. 12, no. 1, pp. 1404-1407, 2005.

[43] E. R. Fossum, "CMOS Image sensors: electronic camera-on-a-chip," IEEE Trans. Electron Devices, vol. 44, no. 10, pp. 1689-1698, Oct. 1997.
[44] H. C. van Assen, M. Egmont-Petersen, and J. H. C. Reiber, "Accurate object localization in gray level images using the center of gravity measure: accuracy versus precision," IEEE Trans. Image Process., vol. 11, no. 12, pp. 1379-1384, Dec. 2002.

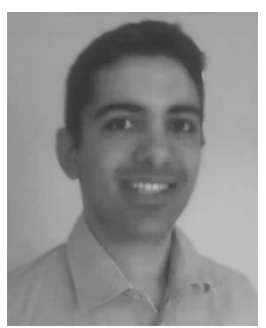

Michael D. Armani received the B.S. degree in mechanical engineering from the University of Maryland, College Park, in 2005.

He joined the Department of Bio-Engineering, University of Maryland, in 2005, where he is currently pursuing the Ph.D. degree. His main interest is in creating engineering technologies that will be used for understanding cancer and aging processes.

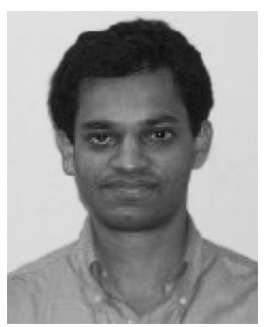

Satej V. Chaudhary was born in Bombay, India, in 1978. He received the B.Tech. degree in aerospace engineering from the Indian Institute of Technology, Bombay, in 2001, the M.S. degree in aerospace engineering from the University of Maryland, College Park, in 2005.

$\mathrm{He}$ is currently pursuing the Ph.D. degree in modeling and control of microfluidic systems at the University of Maryland. His main interest is modeling and control of microfluidic systems.

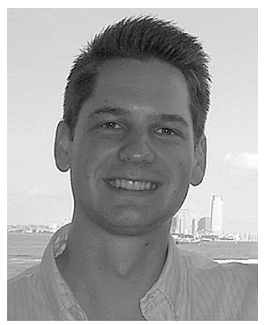

Roland Probst received the Diploma in computer engineering from the University of Applied Sciences, Mannheim, Germany, in 2004.

$\mathrm{He}$ joined the Department of Aerospace Engineering, University of Maryland, College Park, in 2004, where he is currently pursuing the Ph.D. degree. His main interest is in control theory and smart integrated systems, with a primary focus on bio-MEMS applications.

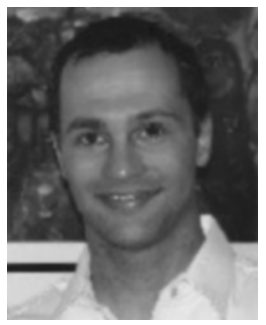

Benjamin Shapiro (M'04) was born in Jerusalem, Israel, in 1973. He received the B.S. degree in aerospace engineering from the Georgia Institute of Technology, Atlanta, in 1995, and the Ph.D. degree in control and dynamical systems from the California Institute of Technology, Pasadena, in 1999.

$\mathrm{He}$ joined the Department of Aerospace Engineering, University of Maryland, College Park, as an Assistant Professor in 2000 and is now an Associate Professor. His main interests are in modeling and control of microsystems, with a primary focus on modeling and control of microfluidic systems for biochemical applications. He is part of the interdisciplinary Small Smart Systems Center and is affiliated with the Bioengineering Graduate Program and the Applied Math And Scientific Computation Program at the University.

Dr. Shapiro was the organizer of the March 2004 NSF workshop on Control and System Integration of Micro- and Nano-Scale Systems and is a recipient of a 2003 NSF CAREER award. 